\title{
Killian-Jamieson Diverticulum Diagnosed as Thyroid Nodule: A Case Report
}

\author{
Lei Huang1, Zhiming Jiang1, Lu He ${ }^{2}$, Lei Su${ }^{1 *}$ \\ ${ }^{1}$ Department of General Surgery, The Affiliated Drum Tower Hospital of Nanjing University Medical School, \\ Nanjing, China \\ ${ }^{2}$ Department of Pathology, The Affiliated Drum Tower Hospital of Nanjing University Medical School, \\ Nanjing, China \\ Email: *suleinjglyy@sina.com
}

How to cite this paper: Huang, L., Jiang, Z.M., He, L. and Su, L. (2018) Killian-Jamieson Diverticulum Diagnosed as Thyroid Nodule: A Case Report. Open Journal of Preventive Medicine, 8, 1-5. https://doi.org/10.4236/ojpm.2018.81001

Received: December 30, 2017

Accepted: January 21, 2018

Published: January 24, 2018

Copyright ( 92018 by authors and Scientific Research Publishing Inc. This work is licensed under the Creative Commons Attribution International License (CC BY 4.0).

http://creativecommons.org/licenses/by/4.0/

\begin{abstract}
Killian-Jamieson diverticulum is a rare type of pharyngoesophageal diverticula. We present a case of Killian-Jamieson diverticulum that mimicked a thyroid nodule under ultrasonography. Fine-needle aspiration was applied to identify the content of the thyroid nodule. However, nothing else but only a few squamous cells, amorphous material, and some inflammatory cells were reported under the microscope. The Killian-Jamieson diverticulum was confirmed during the surgery. There was no complication after the operation at the follow-up visit one month later. Retrospect this case, we find that thyroid ultrasonography combined fine-needle aspiration is a recommended way to increase the sensitivity of detecting hypopharyngeal diverticulum.
\end{abstract}

\section{Keywords}

Pharyngoesophageal Diverticula, Thyroid Nodule, Ultrasonography, Fine-Needle Aspiration

\section{Introduction}

Thyroid diseases are common endocrine disease in clinics. However, symptoms from other neck neoplasms maybe similar to ones from thyroid disease, which cause misdiagnosis [1]. Killian-Jamieson diverticulum is a rare type of pharyngoesophageal diverticula, which can be misdiagnosed as thyroid nodules with ultrasonography [2]. To our knowledge, radiographic presentation of the co-existence of Killian-Jamieson diverticulum and a thyroid tumor has not been previously described. We reported the rare case of 45 -year-old patient with a symptomatic left Killian-Jamieson diverticulum with thyroid nodule goiter. 


\section{Case Report}

A 45-year-old man presented with a suspected thyroid nodule that was detected incidentally on a routine checkup on May 04, 2017. Thyroid ultrasonography (US) revealed a hypoechoic nodule with $2.8 \times 1.6 \mathrm{~cm}$ in the left lobe of thyroid, accompanied with some small ones (Figure 1). A US-guided fine-needle aspiration (FNA) was performed without complication to rule out malignancy. The cytologic examation of the FNA specimen showed only a few squamous cells, amorphous material, and some inflammatory cells, with no evidence of malignancy.

This patient underwent resection of the suspected thyroid nodule. The surgical operation initiated through a collar incision in the middle anterior neck. The subcutaneous tissue and platysma were divided. After separating the omohyoid muscle and the left lobe of thyroid, a mass that seemed to be diverticulum was visualized behind the left lobe of thyroid with definiteboundary (Figure 2). A gastric tube was inserted to identify the mass. After filling some air into the gastric tube, the mass was inflated, which confirmed the diagnosis of a diverticulum. The left lobe of thyroid containing small nodules was removed, then a diverticulotomy was performed. The wound was closed in layers with placement of a closed suction drainage. Histopathological examination showed that thyroidnodules in the left lobe were diagnosed as nodular goiter while the diverticulum was confirmed according to the loss of muscular layer (Figure 3). The patient started on a clear liquid diet on postoperative day 4 . There was no complication after the operation at the follow-up visit one month later.

\section{Discussion}

The esophageal diverticulum is a congenital or acquired circumscribed pouch generated by the herniation of the lining mucous membrane through a defect in

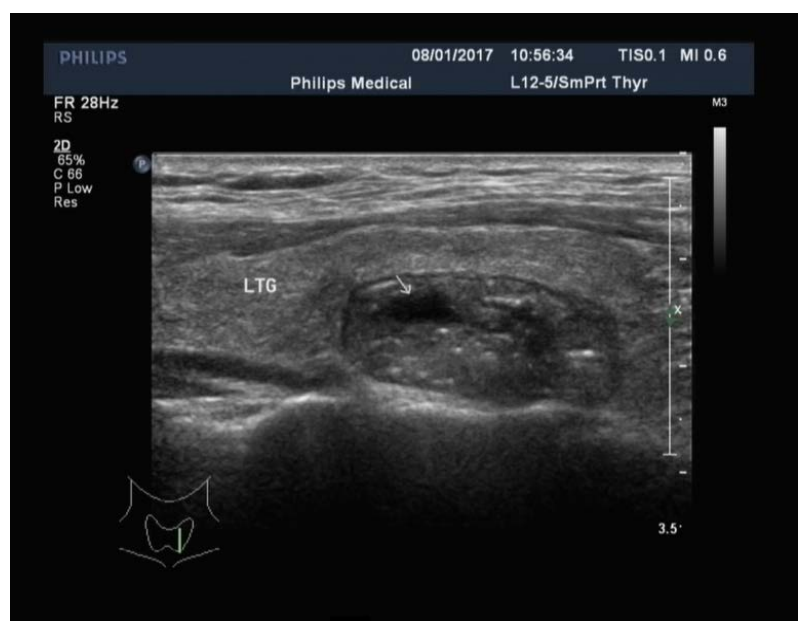

Figure 1. The ultrasonography (US) to the thyroid gland. Thyroid ultrasonography demonstrated a hypoechoic nodule sized $2.8 \times 1.6 \mathrm{~cm}$ in the left lobe of thyroid, accompanied with some smallones. 


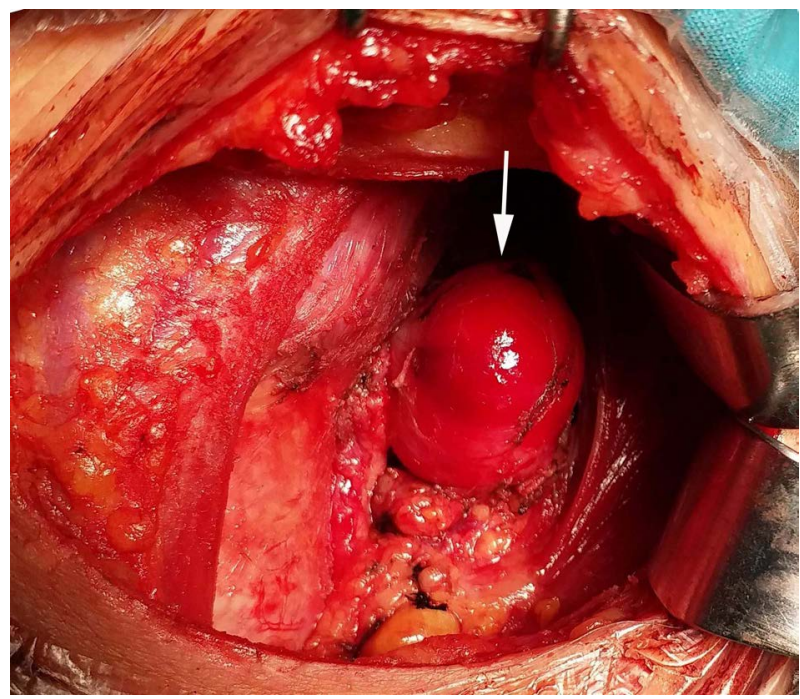

Figure 2. The diverticulum under surgery field. After dividing the omohyoid muscle and the left lobe of thyroid, a mass that seemed to be diverticulum (marked by the arrow) was visualized behind the left lobe of thyroid.

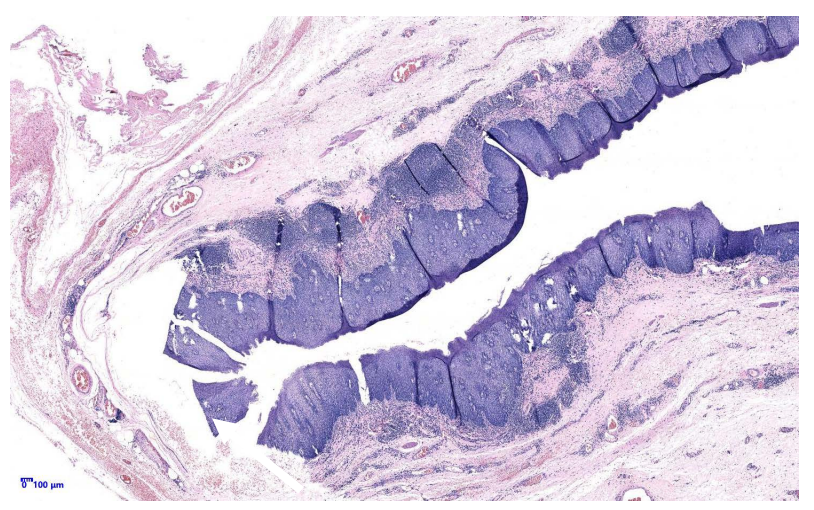

Figure 3. Pathological section of examined specimens. Histopathological examination showed that the diverticulum was confirmed according to the loss of muscular layer.

the esophageal wall [3]. Killian-Jamieson diverticula (KJD) are the rare type of hypopharyngeal diverticula related to the more easily recognized Zenker's diverticulum (ZD). In Rubesin's research, it was reported that 16 patients were diagnosed as Killian-Jamieson diverticulum during a 7-year period while 26 patients were found to be with Zenker's diverticulum in a period of 4 years. Additionally, $75 \%$ of the Killian-Jamieson diverticulua were on the left side and $25 \%$ were bilateral. Moreover, $\mathrm{ZD}$ is more common and symptomatic while KJD is smaller and more likely to be asymptomatic, which resulting in KJD's increased chance to be misdiagnosed as a thyroid nodule. In Rubesin's reporter, $89 \%$ of the patients with KJD showed no symptoms, but for the rest $11 \%$ patient, symptoms including dysphagia, coughing and epigastric pain were the most common ones [4].

US is the most convenient and fastest examination applied to detect neck mass. 
Similarities and differences between pharyngoesophageal diverticulum and the thyroid nodules are concluded in previous reporters. First, air bubbles or other particles, which cause the heterogeneous internal echo, could be regarded as microcalcifications of thyroid cancer, though there is stronger echogenicity and irregularity of the post acoustic shadow. Second, the origin from the digestive tract can be indicated by a hypoechoic rim or a multilayered zone. Third, there is an irregular boundary of the posterior wall of the lesion at the posterior portion of the thyroid gland. Finally, transient changes in size, margins and echogenicity that detected during swallowing or by the compression of the probe suggest the nature of the lesion [5] [6] [7] [8] [9].

When a diverticulum disguises as a thyroid nodule, FNA may be suggested to obtain a pathological diagnosis. There are some studies to confirm the falsenegtive rate of FNA is less than $1 \%$ and false-positive rate is merely $1 \%$ to $3 \%$ in thyroid diagnosis. According to the previous studies, FNA increased the diagnostic accuracy to $87.5 \%$ in total, in which $93.5 \%$ was for benign lesions and 97.3\% was for malignant lesions [10] [11]. However, Li Cao posed the idea that FNA was quite dangerous because the KJ triangle is directly adjacent to the entry point of the recurrent laryngeal nerve into the larynx [12]. This idea is suggestive but complications of FNA have not been reported in such cases so far.

\section{Conclusion}

In conclusion, this study reported a case of a patient who presented with a suspected thyroid nodule in the left lobe of thyroid gland, and underwent a neck exploration surgery. During the surgery, the suspected nodule was proved to be a KJD. Retrospect this case, we find that thyroid US combined FNA is a recommended way to increase the sensitivity of detecting hypopharyngeal diverticulum.

\section{References}

[1] Gray, S.L., O’Neill, G. and McGarry, G. (2014) The Predictive Value of Structured Ultrasonographic Staging for Thyroid Nodules. The Journal of Laryngology \& Otology, 128, 914-921. https://doi.org/10.1017/S0022215114002072

[2] Ekberg, O. and Nylander, G. (1983) Lateral Diverticula from the Pharyngo-Esophageal Junction Area. Radiology, 146, 117-122. https://doi.org/10.1148/radiology.146.1.6401363

[3] Undavia, S., Anand, S.M. and Jacobson, A.S. (2013) Killian-Jamieson Diverticulum: A Case for Open Transcervical Excision. Laryngoscope, 123, 414-417. https://doi.org/10.1002/lary.23639

[4] Rubesin, S.E. and Levine, M.S. (2001) Killian-Jamieson Diverticula: Radiographic Findings in 16 Patients. AJR American Journal of Roentgenology, 177, 85-89. https://doi.org/10.2214/ajr.177.1.1770085

[5] Biggi, E., Derchi, L.E., Cicio, G.R. and Neumaier, C.E. (1982) Sonographic Findings of Zenker's Diverticulum. Journal of Clinical Ultrasound, 10, 395-396. https://doi.org/10.1002/jcu.1870100809

[6] Hayashi, N., Tamaki, N., Konishi, J., Endo, K., Misaki, T., Torizuka, K., et al. (1984) 
Lateral Pharyngoesophageal Diverticulum Simulating Thyroid Adenoma on Sonography. Journal of Clinical Ultrasound, 12, 592-594.

https://doi.org/10.1002/jcu.1870120914

[7] Yahara, T. and Machi, J. (2002) Image of the Month. Zenker Diverticulum. Archives of Surgery, 137, 619-620. https://doi.org/10.1001/archsurg.137.5.619

[8] Kwak, J.Y. and Kim, E.K. (2006) Sonographic Findings of Zenker Diverticula. Journal of Ultrasound in Medicine, 25, 639-642. https://doi.org/10.7863/jum.2006.25.5.639

[9] Jiang, L.X., Hu, B., Wang, Z.G. and Zhao, B.H. (2011) Sonographic Diagnosis Features of Zenker Diverticulum. European Journal of Radiology, 80, e13-e19. https://doi.org/10.1016/j.ejrad.2010.05.028

[10] Ali, S.Z. (2011) Thyroid Cytopathology: Bethesda and Beyond. Acta Cytologica, 55, 4-12. https://doi.org/10.1159/000322365

[11] Gu, P., Zhao, Y.Z., Jiang, L.Y., Zhang, W., Xin, Y. and Han, B.H. (2009) Endobronchial Ultrasound-Guided Transbronchial Needle Aspiration for Staging of Lung Cancer: A Systematic Review and Meta-Analysis. European Journal of Cancer, 45, 1389-1396. https://doi.org/10.1016/j.ejca.2008.11.043

[12] Cao, L., Ge, J., Zhao, D. and Lei, S. (2016) Killian-Jamieson Diverticulum Mimicking a Calcified Thyroid Nodule on Ultrasonography: A Case Report and Literature Review. Oncology Letters, 12, 2742-2745. https://doi.org/10.3892/ol.2016.4984 